\title{
COMPLEJIDAD GRAMATICAL Y NIVELES DE DIFICULTAD EN LECTURAS DE ELE ADAPTADAS Y ORIGINALES
}

\author{
GRAMMATICAL COMPLEXITY AND DIFFICULTY LEVELS IN \\ ADAPTED AND ORIGINAL SPANISH L2 READINGS
}

\author{
IRENE CHECA-GARCIA \\ University of Wyoming. Wyoming, USA \\ ichecaga@uwyo.edu
}

\section{RESUMEN}

Las lecturas de español ELE son clasificadas por las editoriales en varios niveles de dificultad. Sin embargo, poco o ningún criterio se ofrece para su clasificación. Si algún criterio se menciona, éste se refiere a la diversidad de vocabulario, pero no a la complejidad morfosintáctica. Este estudio trata de responder a dos cuestiones fundamentales: a) ¿Hay una relación entre ciertos índices de complejidad morfosintáctica y niveles de dificultad de las lecturas? y b) ¿Difieren los índices de complejidad morfosintáctica de los textos creados originalmente como lecturas ELE y de los que provienen de adaptaciones? En una muestra de 61 fragmentos pertenecientes a 3 niveles diferentes de dificultad (básico, intermedio y avanzado) se analizan los índices primarios de madurez sintáctica y la diversidad verbal. Los resultados muestran que las diferencias entre niveles de dificultad son sólo significativas para los textos originales (aquellos que se crearon originalmente como lecturas ELE vs. textos que son adaptaciones). Además, los niveles intermedio y avanzado se diferencian mucho menos entre sí que con respecto al nivel básico.

Palabras clave: Complejidad sintáctica, lecturas ELE, dificultad de lectura, lectura en L2, adquisición de lenguas extranjeras, español como lengua extranjera.

\section{ABSTRACT}

Spanish L2 readings are classified by editors into different difficulty levels. However, very few criteria if any are mentioned for the classification. When some criterion is mentioned, it is a measure of vocabulary diversity, with no reference to morphosyntactic complexity. The present study seeks to answer two main questions: a) is there a relation between certain indexes of morphosyntactic complexity and the readings levels of difficulty?, and b) are texts created as L2 readings and texts adapted from originally L1 readings different 
with respect to such indexes of morphosyntactic complexity? A sample of 61 fragments belonging to the two different types of texts are classified into 3 different difficulty levels (basic, intermediate and advanced) and are analyzed for primary syntactic maturity indexes and verbal diversity. Results show that only for the texts originally written as L2 readings is the difference between the levels significant. In addition, the intermediate and advanced levels are much less different among them than with respect to the basic level.

Keywords: Syntactic complexity, Spanish L2 readings, reading difficulty, L2 reading, foreign language acquisition, L2 Spanish.

Recibido: 25/01/2013. Aceptado: 16/09/2013.

\section{INTRODUCCIÓN: COMPLEJIDAD MORFOSINTÁCTICA Y DIFICULTAD DE LAS LECTURAS ELE}

$\square$ s frecuente suplementar los materiales de clase de una lengua extranjera con Clecturas de cuentos breves. La necesidad de una gradación de los diferentes tipos de input es apoyada por teorías como la del Input Comprensible, o “i+1" (Krashen, 1982) o por la Teoría de la Acomodación Comunicativa (Giles y Ogay, 2007). Para que el aprendizaje sea posible, y también la comunicación en general, el input que recibe el oyente o el lector debe de estar dentro de sus posibilidades de comprensión, o sólo un nivel por encima. Diversas investigaciones empíricas apoyan esta decisión pedagógica, mostrando que los textos con una mayor simplicidad son comprendidos mejor y por tanto podrían estimular más el aprendizaje (cfr. Jeong, 1987; Lee, 1998; Kim, 1985, y Choi, 1996, entre otros muchos). Otras investigaciones señalan que esta ayuda podría ocurrir sólo en los niveles más bajos de competencia del aprendiz (Blau, 1982; Brown, 1987; Chaudron, 1983; Long, 1985). Al margen de los resultados de investigaciones varias, las lecturas con sintaxis y vocabulario modificados son de hecho empleadas en la instrucción del español como L2, tanto en libros de texto -generalmente fragmentos de obras para L1 simplificados- como en libros que consisten en colecciones de lecturas graduadas o en una única lectura graduada. Se necesita entonces determinar si la gradación por niveles de las lecturas se da realmente y en qué términos. Un primer estudio, muy elemental ${ }^{1}$, fue llevado a cabo por Gaies (1979) para el caso del inglés. No se hizo un tratamiento estadístico de los datos, pero se puede observar una tendencia a que la gradación se relacione con la complejidad sintáctica, sobre todo en el nivel básico. Pero, ¿qué ocurre en el caso del español?

${ }^{1}$ Este estudio no controló la variable Modo de discurso (véase la sección de metodología de nuestro estudio) y sólo empleó un índice en su estudio: la Longitud de la Unidad Terminal (véase el apartado 2.2. para una definición del índice). Además, sólo 26 fragmentos fueron analizados y éstos incluyeron tanto lecturas extras como fragmentos dentro de un libro de texto. 
Las lecturas ELE son organizadas por la editorial y dentro de la colección en que aparecen en niveles de dificultad. Pero a pesar de que hay creados estándares para los diferentes niveles de competencia en una lengua extranjera (los estándares de la ACTFL en EE.UU. o los niveles del MCERL, el Marco Común Europeo de Referencia para las Lenguas), las editoriales apenas los utilizan -en algunos pocos casos mencionan el MCER-y tampoco tienen unos estándares propios explícitos. En algunas colecciones se especifica la diversidad del vocabulario en número de palabras, mientras que en otras ni tan siquiera esto.

En consecuencia, en las lecturas breves para aprendices de ELE no aparecen muchas indicaciones de lo que se ha tenido en cuenta para cada nivel, y la única medida específica mencionada -cuando hay alguna- se refiere a la variedad del vocabulario. Medidas de elaboración sintáctica o morfológica no aparecen explícitamente. Sin embargo, medidas de complejidad sintáctica o de cohesión, por ejemplo, han demostrado estar correlacionadas con el éxito en la comprensión de determinados textos para hablantes no nativos de inglés. Ya en 1967 MacNamara probó que la rapidez de lectura tanto para L1 como para L2 estaba afectada por la similaridad del orden de elementos al orden de elementos estándar en la lengua. Barry y Lazarte (1995) mostraron cómo la tasa de recuerdo del contenido depende en parte de la posición de dicho contenido en la oración principal, subordinada de primer orden o subordinada de segundo orden. Los resultados de Oh (2001) también muestran que la simplificación del texto ayuda a comprenderlo mejor, puesto que los estudiantes que leyeron el texto simplificado lo comprendieron mejor que los que leyeron el elaborado y que los que leyeron el texto original. Barnett (1986) encontró que la simplificación morfosintáctica afectaba al éxito de la comprensión tanto como la simplificación de vocabulario, y que ninguna de estas simplificaciones ayudaban a la comprensión si los aprendices no tenían un nivel aceptable en la otra habilidad. El estudio de Lee (1998), centrado únicamente en el subjuntivo, también señaló que la mayor dificultad morfológica afecta asimismo al éxito de la comprensión del texto. Cabe preguntarse entonces si esta falta de atención a lo morfosintáctico para determinar el nivel de dificultad ocurre de facto al escribir estas lecturas, o si, por el contrario, aunque no se mencione explícitamente, las lecturas presentan diferente elaboración morfosintáctica según el nivel de dificultad en el que se han clasificado.

Además, esta elaboración sintáctica según los niveles de dificultad podría ser diferente según el tipo de texto, dependiendo de si fue escrito originalmente como un texto más sencillo para los aprendices de ELE (lecturas originales), o es una adaptación/simplificación de un texto originalmente escrito para hablantes nativos de español (lecturas adaptadas). No hay apenas estudios en español sobre este particular, con la excepción de un estudio piloto de Checa-Garcia (2005b). En inglés se realizó un estudio similar (Crossley, Louwerse, McCarthy y McNamara, 2007) para describir las diferencias lingüísticas encontradas entre textos originales 
para hablantes nativos y textos elaborados para hablantes no nativos, encontrándose algunas diferencias significativas.

En el presente trabajo nos proponemos centrarnos en la complejidad sintáctica -entre las diversas posibles caracterizaciones- de los textos creados para hablantes no nativos, sea por simplificación de un texto originalmente escrito para hablantes nativos. Se quiere comprobar si los grados de dificultad en que están clasificadas las lecturas revelan un grado de complejidad morfosintáctica mayor, y también si hay diferencias entre los dos tipos de textos: lecturas originales vs. adaptadas.

\section{METODOLOGÍA}

\subsection{Corpus y clasificación de los textos}

Se elaboró un corpus de 61 fragmentos con 220 a 300 palabras, con un total de 1.484 oraciones $^{2}$. Todos los fragmentos fueron fragmentos narrativos para controlar la variable "modo del discurso", probada influyente en diversas medidas de variación sintáctica (Crowhurst y Piche, 1979; Crowhurst, 1980; Prater y Padia, 1983 para el inglés; Véliz de Vos, 1999 y Espinet de Jesús, 1996 para el español). Los fragmentos se extrajeron de libros de lectura ELE publicados en diferentes colecciones. Por un lado, se seleccionaron libros de lecturas graduadas escritas ex profeso para estudiantes de ELE (lecturas originales) clasificados por sus respectivas editoriales en diferentes niveles. Por otro, se seleccionaron libros de lecturas graduadas que provienen de adaptaciones para el estudiante de ELE de obras literarias originales, novelas y cuentos fundamentalmente (lecturas adaptadas).

Para poder determinar si hay una relación entre los niveles de dificultad de las lecturas de ELE y la elaboración de su morfología y sintaxis fue necesario identificar los niveles de dificultad en que las obras son clasificadas. Esta identificación resultó tarea complicada debido a la heterogeneidad de las clasificaciones usadas por las diferentes editoriales. Estas clasifican sus materiales de lectura en desde 4 hasta 6 niveles. Tampoco es posible determinar la equivalencia de niveles entre las distintas colecciones, ni siquiera atendiendo a la diversidad de vocabulario, puesto que algunas colecciones indican tal diversidad y otras no. Decidimos finalmente considerar tres niveles de dificultad, siguiendo el marco europeo de referencia para la enseñanza de lenguas extranjeras, MCERL. En este marco se habla de tres niveles: básico (A), intermedio (B) y avanzado (C), cada uno de los cuales está divido en dos niveles a su vez, por ejemplo A1 y A 2. Además, la mayoría de las editoriales clasifican este tipo de lecturas en tres niveles: básico, intermedio y avanzado, con

${ }^{2}$ Unidades terminales en realidad, véase el siguiente apartado, 2.2., para una definición de esta unidad. 
algunas subclasificaciones en uno o más de los niveles. Además, en un estudio piloto con un corpus más pequeño Checa-Garcia (2005b) comprobó que sólo una división entre tres niveles reflejaba diferencias significativas entre los niveles ${ }^{3}$.

Las variables independientes fueron por tanto dos: los tres niveles de dificultad de las obras, y si éstas fueron lecturas originales o adaptadas. El número de ejemplares para cada tipo de texto y cada nivel quedó distribuido conforme se muestra en la Tabla I:

Tabla I. Distribución de fragmentos por tipo de texto y nivel.

\begin{tabular}{|l|r|r|r|r|r|r|r|r|}
\hline \multirow{2}{*}{ Tipo de texto } & \multicolumn{9}{|l|}{ Nivel de dificultad } \\
\cline { 2 - 9 } & \multicolumn{1}{|c|}{ Básico } & \multicolumn{2}{|l|}{ 2 Intermedio } & \multicolumn{2}{l|}{ 3 Avanzado } & \multicolumn{2}{l|}{ Total } \\
\hline Original & 14 & $23.0 \%$ & 19 & $31.1 \%$ & 11 & $18.0 \%$ & 44 & $72.1 \%$ \\
\hline Adaptado & 4 & $6.6 \%$ & 6 & $9.8 \%$ & 7 & $11.5 \%$ & 17 & $27.9 \%$ \\
\hline Total & 18 & $29.5 \%$ & 25 & $41.0 \%$ & 18 & $29.5 \%$ & 61 & $100.0 \%$ \\
\hline
\end{tabular}

\subsection{Medidas de la complejidad morfosintáctica}

En cuanto a las variables para medir la elaboración o complejidad morfosintáctica, se utilizó cuatro. Tres de ellas provienen de los trabajos sobre madurez sintáctica de K.W. Hunt (1965, 1967, 1970a, 1970b): Longitud de la unidad terminal, Longitud de la cláusula, e Índice de subordinación. La cuarta, Diversidad de las formas verbales (en adelante Diversidad verbal), la creamos para este estudio para incluir el aspecto morfológico, ya que los tres primeros índices son sólo sintácticos ${ }^{4}$.

Los índices de madurez sintáctica fueron diseñados para medir el progreso en la escritura de L1. Su crecimiento conforme los productores avanzan en edad resultó constante y significativo en varios estudios en diversas lenguas, tanto en estudios longitudinales (Loban, 1976; Rodrigues, 1981) como en estudios que comparan diferentes edades (por ejemplo en Hunt, 1967, 1970a; Rodríguez Fonseca, 1991; Torres González 1996; Nippold, 1998; Checa-Garcia, 2005a). También se han empleado para medir el progreso en la producción escrita de L2

\footnotetext{
${ }^{3}$ Este estudio incluyó los tres índices primarios de Hunt (1965), pero no usó índice morfológico alguno.

${ }^{4}$ Cada fragmento fue etiquetado conforme a: colección, nivel asignado por la editorial, nivel reasignado por este estudio y tipo de lectura (original vs. adaptada). A continuación se añadieron etiquetas al final de cada unidad de análisis (unidad terminal y cláusula) y de cada una de las formas verbales presentes en los fragmentos. Seguidamente, se trató el corpus con el lenguaje R, con el que se extrajo una tabla de frecuencias de unidades terminales, cláusulas y número de formas verbales totales (ejemplares) y diferentes (tipos) por fragmento. Estos datos, junto con los correspondientes a tipo de lectura y nivel de la lectura por fragmento, fueron trasladados a SPSS para su exploración y tratamiento estadístico (véase el apartado 3.1.).
} 
(Larsen-Freeman y Strom, 1977; Larsen-Freeman, 1978; Flahive y Snow 1980; Gaies, 1980, y Mendelsohn, 1983, para una reflexión al respecto), si bien su aplicación en este caso ha requerido "unidades terminales libres de errores" y la definición de qué cuenta como un error y qué no ha sido polémica. En nuestro estudio sin embargo, usamos los índices primarios de Hunt para medir input escrito por hablantes nativos y, por tanto, producción de L1, aunque de ésta interese su influencia en la comprensión por parte de hablantes de español como L2. Varios estudios previos usaron estos índices para medir la complejidad sintáctica en adultos nativos (Espinet de Jesús, 1992; Torres González, 1997; Hunt, 1970a), si bien no como medida indirecta de complejidad sintáctica potencialmente influyente en la comprensión de una L2.

La Longitud de la unidad terminal (en adelante: Longitud de la UT) consiste en el número de palabras por unidad terminal (UT), siendo la UT: "one main clause plus any subordinate clause or nonclausal structure that is attached to or embeded in it" (Hunt, 1970a: 4). Es decir, para calcular la Longitud de la UT se recuentan todas las palabras del fragmento, número que se divide por el total de UTs. Estas consisten en un verbo principal y cualquier sintagma o cláusula dependiente de él.

La Longitud de la cláusula es el número de palabras por cláusula, que a su vez consiste en "any expression containing a subject or coordinated subjects and a finite predicate or coordinate predicates" (Hunt, 1970a: 4). Se entiende por cláusula cualquier verbo conjugado con sintagmas dependientes de él y algunas formas no personales del verbo5.

El Índice de subordinación es el número de cláusulas por unidad terminal. Se obtiene tras dividir el número de cláusulas en el fragmento por el número de UTs.

Por tanto, la Longitud de la unidad terminal es igual a la Longitud de la cláusula por el Índice de subordinación (número de cláusulas por unidad terminal).

Finalmente, la Diversidad de formas verbales o Diversidad verbal es el número total de formas verbales conjugadas divido por el número de tipos de formas conjugadas presentes en el texto. Esta es una variable independiente de las anteriores, aunque cuanto mayor es el número de cláusulas, más probable es que este índice de diversidad de las formas verbales aumente. Hemos incluido esta medida porque

\footnotetext{
${ }^{5}$ Seguimos la fragmentación estipulada en Checa-Garcia (2005a) en el capítulo 7 sobre segmentación (especialmente pp. 404-445). No podemos reproducir aquí todos los detalles por razones de espacio. Baste recordar el resumen de su discusión sobre el tratamiento de las formas no personales:

"en la controvertida decisión de cómo tener en cuenta las formas no personales del verbo, nos decantamos por considerarlos cláusulas (...) también cuando no incorporan a su predicación adyacente alguno. Excluimos de la consideración de cláusulas a las perífrasis, por entender que se trata de formas peculiares del verbo cuyo estudio ha de incluirse en un nivel sintagmático o incluso morfológico, así como a las formas no personales del verbo que puedan aparecer en las construcciones absolutas, que tampoco constituyen cláusulas a nuestro entender, sino adyacentes oracionales" (415).
} 
una de las maneras más habituales de secuencializar la enseñanza del español es mediante las formas verbales, enseñándose primero el presente, luego el pretérito, después el imperfecto, futuro y condicional, las formas compuestas, las formas del subjuntivo, etc. Por tanto, cuánto mayor es el número de formas diferentes verbales, mayor debería ser considerado el nivel de dificultad.

Las ventajas de usar estos índices son que: (i) al ser cuantitativos permiten pruebas de significatividad y su determinación resulta objetiva; (ii) en el caso de los índices primarios de Hunt, por haberse usado en anteriores estudios permiten comparaciones con estudios de producción en L1 y L2; y, finalmente, (iii) podrían en un futuro ser utilizados para comparar la complejidad de la producción con efectos de ésta en la comprensión.

\subsection{Preguntas e hipótesis del estudio}

Nuestras dos preguntas principales son: ¿Están relacionados estos índices de elaboración morfosintáctica con los niveles de dificultad en que se clasifican las lecturas de ELE? Y, ¿̇ocurre esta relación por igual en los dos tipos de lecturas ELE? Partimos de la hipótesis de que tales relaciones existen y de que son significativamente diferentes para los dos tipos de texto, por tanto, nuestras hipótesis nulas a rechazar son:

H0a. Los índices de elaboración morfosintáctica usados no se correlacionan con los niveles de dificultad en que están clasificadas las lecturas de ELE.

H0b. Los índices de elaboración morfosintáctica se comportan de la misma manera en los textos originales que en los adaptados.

\section{RESULTADOS}

\subsection{Exposición del análisis efectuado}

Primero se expondrá la variación observada en los cuatro índices (variables dependientes) según la primera variable independiente, Nivel de dificultad (apartado 3.2.) y después los resultados según la variable independiente Tipo de lectura (apartado 3.3.). En tercer lugar se incluye un estudio estadístico del cruce de las dos variables independientes (apartado 3.4.). El apartado de resultados finaliza con un estudio de la correlación entre los índices de complejidad morfosintáctica (apartado 3.5.). 


\subsection{Influencia de los niveles de dificultad sobre los índices de elaboración morfosintáctica}

La Tabla II muestra principales valores descriptivos de cada índice de elaboración morfosintáctica según el Nivel de dificultad. Las figuras 1 a 4 muestran los valores medios de cada índice para cada uno de los niveles de dificultad.

Tabla II. Estadísticos descriptivos (tendencia central y dispersión) de los índices de elaboración morfosintáctica por Nivel de dificultad de los fragmentos.

\begin{tabular}{|l|l|r|r|r|r|r|}
\hline & Dificultad & Media & Mediana & \multicolumn{1}{c|}{ Mín. } & \multicolumn{1}{c|}{ Máx. } & Desviac. Std. \\
\hline \multirow{4}{*}{$\begin{array}{l}\text { Longitud de } \\
\text { la UT }\end{array}$} & Nivel 1 & 9,13 & 8,66 & 6,24 & 13,17 & 2,01 \\
\cline { 2 - 7 } & Nivel 2 & 13,15 & 11,70 & 6,43 & 24,00 & 4,48 \\
\cline { 2 - 7 } & Nivel 3 & 13,29 & 12,41 & 8,71 & 23,40 & 3,83 \\
\cline { 2 - 7 } & Total & 12,00 & 11,19 & 6,24 & 24,00 & 4,11 \\
\hline \multirow{4}{*}{$\begin{array}{l}\text { Índice de } \\
\text { subordinación }\end{array}$} & Nivel 1 & 1,64 & 1,61 & 1,22 & 2,47 &, 32 \\
\cline { 2 - 7 } & Nivel 2 & 2,04 & 1,84 & 1,28 & 5,15 &, 71 \\
\cline { 2 - 7 } & Nivel 3 & 2,13 & 2,07 & 1,38 & 3,21 &, 54 \\
\cline { 2 - 7 } & Total & 1,95 & 1,81 & 1,22 & 5,15 &, 60 \\
\hline \multirow{4}{*}{\begin{tabular}{l} 
Longitud de cláusula \\
\cline { 2 - 7 }
\end{tabular}} & Nivel 1 & 6,05 & 5,92 & 4,73 & 7,20 &, 72 \\
\cline { 2 - 7 } & Nivel 2 & 6,98 & 6,67 & 4,08 & 9,54 & 1,44 \\
\cline { 2 - 7 } & Nivel 3 & 6,71 & 6,81 & 4,61 & 8,78 & 1,08 \\
\cline { 2 - 7 } & Total & 6,63 & 6,55 & 4,08 & 9,54 & 1,21 \\
\hline \multirow{4}{*}{$\begin{array}{l}\text { Diversidad } \\
\text { verbal }\end{array}$} & Nivel 1 & 0.1266 & 0.1869 & 0.1524 & 0.2305 & 0.0231 \\
\cline { 2 - 7 } & Nivel 2 & 0.1646 & 0.1624 & 0.1026 & 0.2601 & 0.0350 \\
\cline { 2 - 7 } & Nivel 3 & 0.1684 & 0.1533 & 0.1624 & 0.2133 & 0.0258 \\
\cline { 2 - 7 } & Total & 0.1677 & 0.1624 & 0.1025 & 0.2601 & 0.0303 \\
\hline
\end{tabular}

Las figuras 1 a 4 muestran el comportamiento de cada uno de los índices en cada nivel de dificultad y el nivel de significatividad alcanzado ${ }^{6}$.

${ }^{6}$ Siempre que la variable dependiente cumplía las condiciones necesarias se aplicó el test paramétrico ANOVA. Cuando ello no fue posible, se aplicó el test no paramétrico de la $\chi 2$, lo que se señala con un * junto a la significatividad de diferencias alcanzada en el test. 
Figura 1. Comportamiento de la Longitud de la UT en los tres niveles de dificultad.

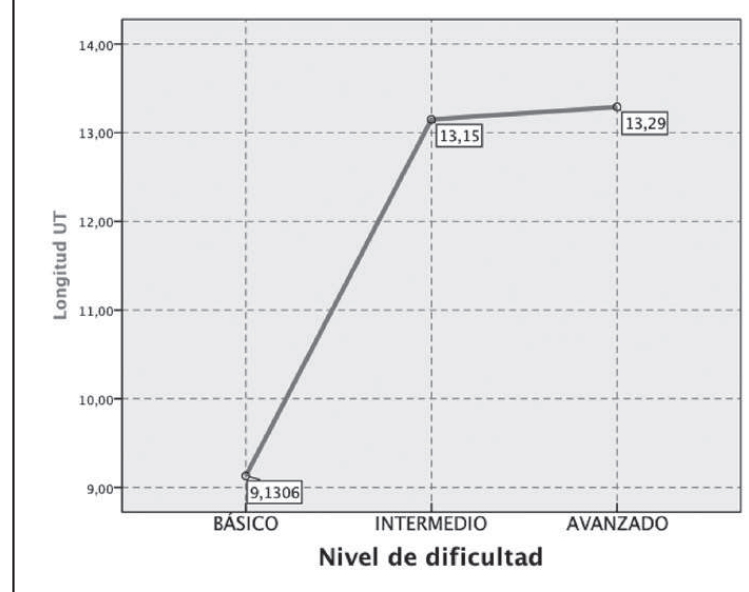

Figura 3. Comportamiento del Índice de subordinación en los tres niveles de dificultad.

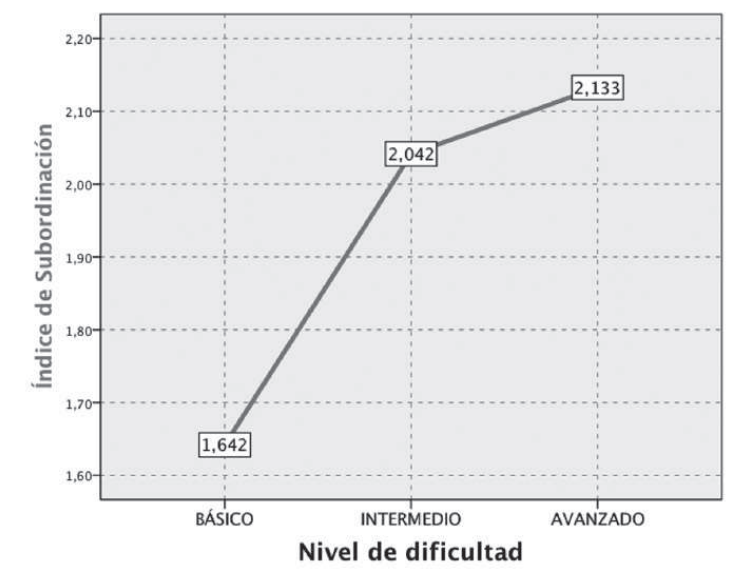

Figura 2. Comportamiento de la Longitud de la cláusula en los tres niveles de dificultad.

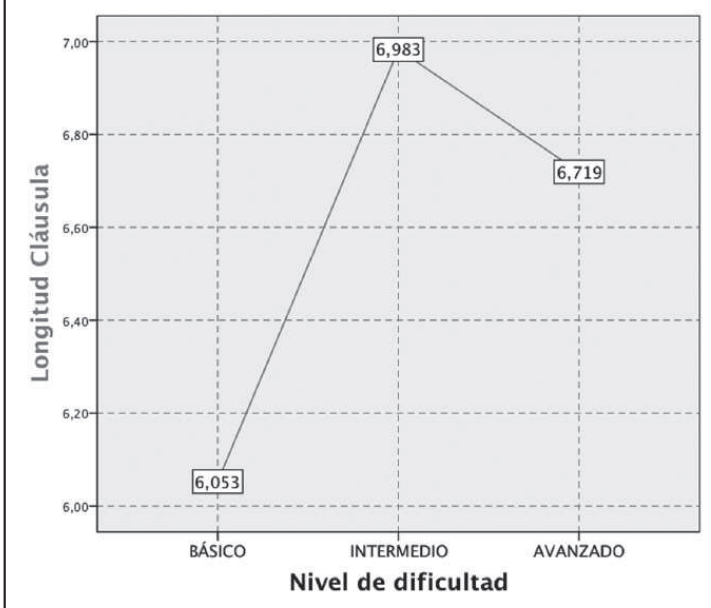

Figura 4. Comportamiento del Índice de diversidad verbal en los tres niveles de dificultad.

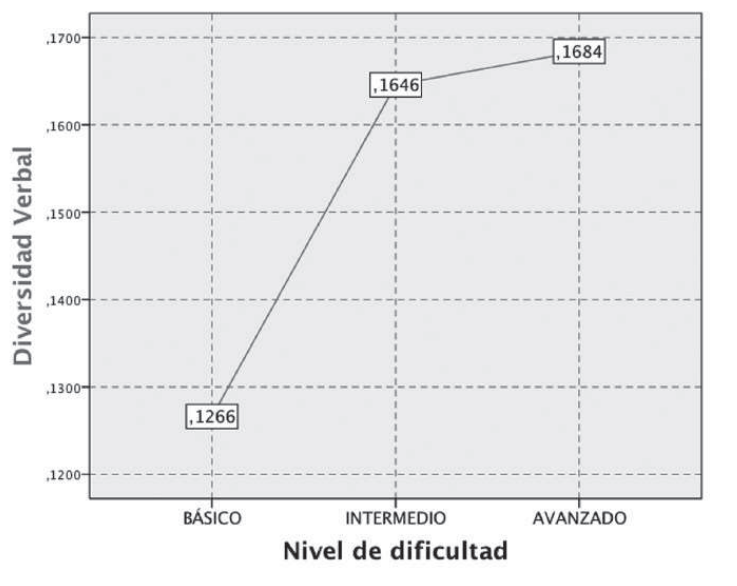

Como se puede observar tanto en la Tabla II como en las figuras 1 a 4 , hay una tendencia de todos los índices, salvo la Longitud de la cláusula, a aumentar conforme aumenta el nivel de dificultad asignado a los textos. Este aumento es mayor entre el nivel básico y el intermedio que entre el intermedio y el avanzado. En el caso de la Longitud de la cláusula, aunque ésta aumenta entre el nivel básico y el avanzado como ocurre con el resto de los índices, decrece entre el nivel intermedio y el avanzado. No obstante, ¿`son estas diferencias estadísticamente significativas? La Tabla III resume los resultados de aplicar tests estadísticos de la varianza. Siempre que la distribución de la variable dependiente en cuestión cumplía las condiciones necesarias ${ }^{7}$ se aplicó el test paramétrico ANOVA. Cuando ello no fue

\footnotetext{
${ }^{7}$ Se asumió la independencia de los valores de las variables dependientes. La distribución de las
} 
posible, se aplicó el test no paramétrico de la $\mathrm{X}^{2}$, lo que se señala con un * junto a la significatividad de diferencias alcanzadas en el test. Aquellas diferencias que sí resultaron estadísticamente significativas son resaltadas en negrita ${ }^{8}$.

Tabla III. Significatividad de diferencias (valores p) de los índices de complejidad morfosintáctica según el Nivel de dificultad.

\begin{tabular}{|c|c|c|c|c|}
\hline Índices & $\begin{array}{c}\text { Longitud } \\
\text { de la UT }\end{array}$ & $\begin{array}{c}\text { Longitud } \\
\text { de la cláusula }\end{array}$ & $\begin{array}{c}\text { Índice de } \\
\text { Subordinación }\end{array}$ & $\begin{array}{c}\text { Diversidad } \\
\text { Verbal }\end{array}$ \\
\hline Signf. & $\mathbf{0 , 0 0 1}$ & 0,069 & $\mathbf{0 , 0 0 5 *}$ & $\mathbf{0 , 0 5}^{*}$ \\
\hline
\end{tabular}

Todas las variables resultaron significativamente relacionadas con los niveles de dificultad excepto la Longitud de la cláusula. Longitud de la UT e Índice de subordinación cambian muy significativamente, mientras la Diversidad verbal lo hace significativamente sólo. Queda por tanto rechazada nuestra hipótesis nula H0a y confirmadas nuestras sospechas excepto para la Longitud de la cláusula: sí hay cierta correlación entre los niveles de dificultad y la complejidad morfosintáctica de los textos.

Para comprobar si cuando las diferencias son significativas éstas los son entre todos los niveles de la variable independiente o sólo entre algunos de ellos se realizó una prueba de Scheffé, que agrupa niveles de la variable independiente que no son significativamente diferentes (Tabla IV). Sólo encontramos agrupaciones de los niveles para la Longitud de la UT y para la Diversidad verbal, en las que se distinguen el nivel básico frente a intermedio y avanzado. En el caso del Índice de subordinación, las diferencias entre el nivel básico y el avanzado son significativas, pero no entre el intermedio con ninguno de estos niveles.

Tabla IV. Pruebas de Scheffé para los 4 índices según Nivel de dificultad.

\begin{tabular}{|l|l|}
\hline Índice & Agrupación \\
\hline Longitud de la UT & Básico vs Intermedio\&Avanzado \\
\hline Longitud de la cláusula & No hay grupos \\
\hline Subordinación & Intermedio puede formar grupo con Básico o con Avanzado \\
\hline Diversidad verbal & Básico vs. Intermedio/Avanzado \\
\hline
\end{tabular}

medias entre los niveles de las variables independientes fue testada para las condiciones de los tests paramétricos: normalidad y homocedasticidad. No se trató de normalizar los valores de los índices.

${ }^{8}$ Los mismos procedimientos y convenciones se aplican al resto de tablas de significatividad del presente estudio. 


\subsection{Diferencias entre los textos originales y los adaptados en su elaboración sintáctica}

Las medidas de tendencia central y dispersión para cada índice según el tipo de texto, originalmente escrito como texto para aprendices de L2, o adaptado de textos para hablantes nativos, pueden encontrarse en la Tabla V. Las figuras 5 a 8 reflejan las diferentes medias para cada índice según el Tipo de texto.

Tabla V. Medias de los índices de elaboración morfosintáctica según el tipo de texto.

\begin{tabular}{|l|l|r|r|r|r|r|}
\hline \multirow{2}{*}{ Índice } & Tipo texto & Media & Mediana & Mínimo & Máximo & \multicolumn{1}{c|}{$\begin{array}{c}\text { Desviac. } \\
\text { Std. }\end{array}$} \\
\hline \multirow{2}{*}{$\begin{array}{l}\text { Longitud } \\
\text { UT }\end{array}$} & Original & 10,84 & 10,15 & 6,24 & 21,27 & 3,30881 \\
\cline { 2 - 7 } & Adaptado & 15,03 & 13,81 & 9,77 & 24,00 & 4,53037 \\
\cline { 2 - 7 } & Total & 12,00 & 11,19 & 6,24 & 24,00 & 4,11160 \\
\hline \multirow{2}{*}{$\begin{array}{l}\text { Índice de } \\
\text { Subord. }\end{array}$} & Original & 1,74 & 1,76 & 1,23 & 2,50 &, 29332 \\
\cline { 2 - 7 } & Adaptado & 2,49 & 2,30 & 1,59 & 5,15 &, 83393 \\
\cline { 2 - 7 } & Total & 1,95 & 1,81 & 1,23 & 5,15 &, 60125 \\
\hline \multirow{2}{*}{$\begin{array}{l}\text { Longitud } \\
\text { Cláusula }\end{array}$} & Original & 6,66 & 6,52 & 4,09 & 9,55 & 1,25954 \\
\cline { 2 - 7 } & Adaptado & 6,57 & 6,68 & 4,62 & 8,66 & 1,11720 \\
\cline { 2 - 7 } & Total & 6,63 & 6,55 & 4,09 & 9,55 & 1,21303 \\
\hline \multirow{2}{*}{$\begin{array}{l}\text { Diversidad } \\
\text { Verbal }\end{array}$} & Original &, 15 &, 14 &, 06 &, 26 &, 0467083 \\
\cline { 2 - 7 } & Adaptado &, 18 &, 19 &, 09 &, 26 &, 0493539 \\
\cline { 2 - 7 } & Total &, 15 &, 15 &, 06 &, 26 &, 0492446 \\
\hline
\end{tabular}

Figura 5. Diferencias en la Longitud de la unidad terminal entre textos originales y adaptados.

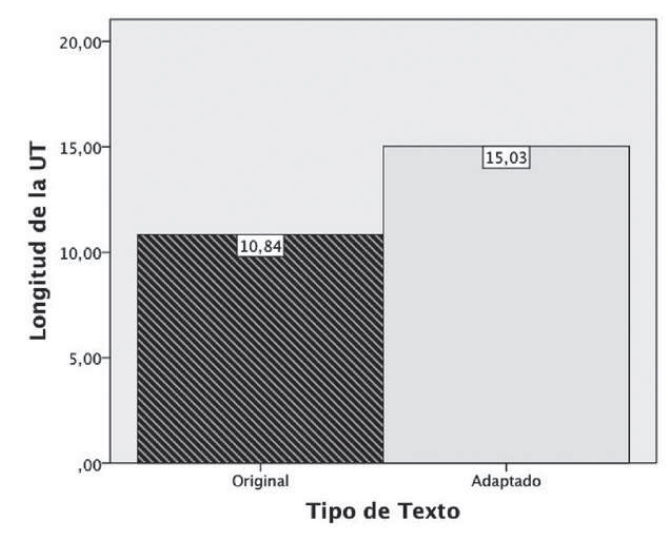

Figura 6. Diferencias en la Longitud de la Cláusula entre textos originales y adaptados.

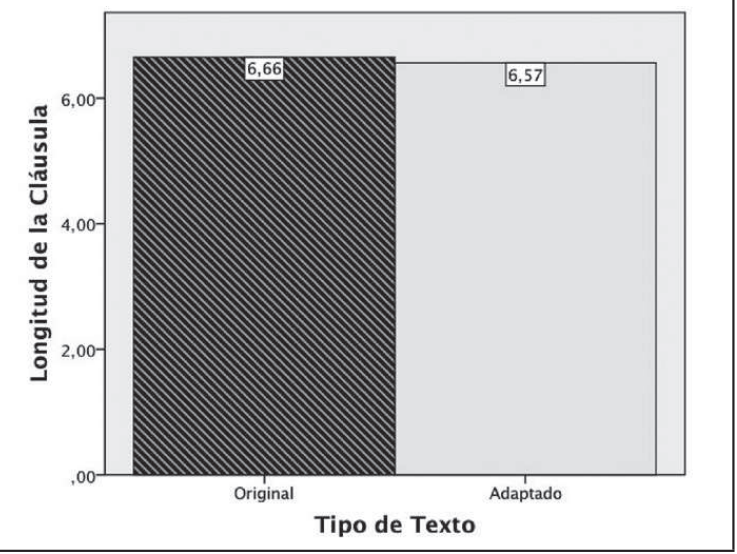


Figura 7. Diferencias en el Índice de subordinación entre textos originales y adaptados.

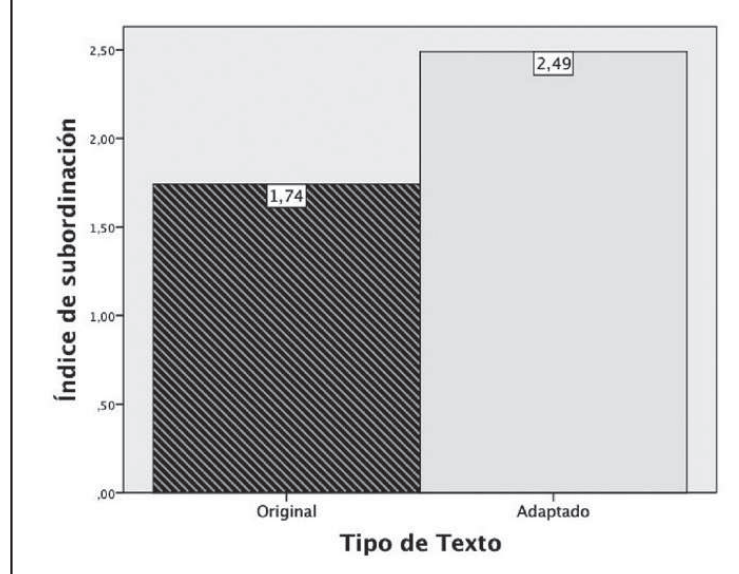

Figura 8. Diferencias en el Índice de diversidad verbal entre textos originales y adaptados.

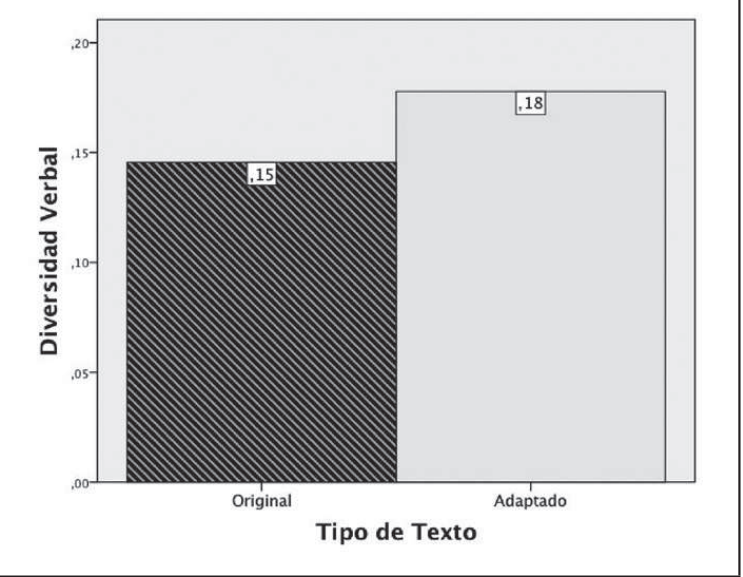

En todos los casos, los índices se relacionaron con el tipo de texto en la misma dirección: los textos adaptados siempre muestran más elaboración sintáctica que los originales, salvo en el caso, de nuevo, de la Longitud de la cláusula, en la que los textos originales muestran una mayor complejidad muy ligeramente $(6,66$ frente a 6,57). Veamos ahora si estas diferencias son significativas en la Tabla VI.

Tabla VI. Significatividad de diferencias (valores p) de los índices según el Tipo de texto.

\begin{tabular}{|c|c|c|c|c|}
\hline Índices & $\begin{array}{c}\text { Longitud } \\
\text { de la UT }\end{array}$ & $\begin{array}{c}\text { Longitud } \\
\text { de la cláusula }\end{array}$ & $\begin{array}{c}\text { Índice de } \\
\text { Subordinación }\end{array}$ & $\begin{array}{c}\text { Diversidad } \\
\text { Verbal }\end{array}$ \\
\hline Signf. & $\mathbf{0 , 0 0 0 1}$ & 0,846 & $\mathbf{0 , 0 0 0 1}^{*}$ & $\mathbf{0 , 0 2 2}^{*}$ \\
\hline
\end{tabular}

Todos los índices de elaboración morfosintáctica estuvieron relacionados con el Tipo de texto significativamente, a excepción de nuevo de la Longitud de la cláusula. Entre los índices relacionados significativamente, otra vez Longitud de la UT e Índice de Subordinación se relacionaron de forma muy altamente significativa, mientras que la Diversidad Verbal se relacionó significativamente también, pero en menor medida estadísticamente. Se rechazó por tanto la hipótesis nula Hob con la excepción de la Longitud de la cláusula. 


\subsection{Variación de los índices de complejidad morfosintácticos según Nivel de dificultad por Tipo de texto}

Comprobado que los textos adaptados para lectura ELE tienen en general una mayor complejidad morfosintáctica que los textos escritos originalmente como lectura ELE, a excepción de la Longitud de la cláusula, cabe preguntarse cómo se comportan los índices por niveles en cada tipo de texto, es decir: ¿presentan los mismos crecimientos o decrecimientos en complejidad morfosintáctica los textos originales y los adaptados? La tabla VII muestra los valores medios para cada uno de los índices de elaboración morfosintáctica por Tipo de texto y Nivel de dificultad.

Tabla VII. Índices de elaboración morfosintáctica por Nivel de dificultad y Tipo de texto.

\begin{tabular}{|l|l|r|r|r|r|}
\hline \multirow{4}{*}{ Índices } & \multirow{2}{*}{ Tipo de texto } & \multicolumn{4}{|c|}{ Niveles de dificultad } \\
\cline { 3 - 6 } & & \multicolumn{4}{|c|}{} \\
\cline { 2 - 6 } & & Básico & Intermedio & Avanzado & \multicolumn{1}{c|}{ Total } \\
\hline \multirow{3}{*}{$\begin{array}{l}\text { Longitud de la } \\
\text { UT }\end{array}$} & Original & 8,33 & 12,48 & 11,19 & 10,83 \\
\cline { 2 - 6 } & Adaptado & 11,95 & 15,26 & 16,58 & 15,02 \\
\cline { 2 - 6 } & Total & 9,13 & 13,15 & 13,29 & 12,00 \\
\hline \multirow{3}{*}{$\begin{array}{l}\text { Índice de } \\
\text { Subord. }\end{array}$} & Original & 1,54 & 1,86 & 1,80 & 1,74 \\
\cline { 2 - 6 } & Adaptado & 1,99 & 2,62 & 2,66 & 2,49 \\
\cline { 2 - 6 } & Total & 1,64 & 2,04 & 2,13 & 1,95 \\
\hline \multirow{2}{*}{$\begin{array}{l}\text { Longitud de la } \\
\text { Cláusula }\end{array}$} & Original & 5,88 & 7,17 & 6,75 & 6,65 \\
\cline { 2 - 6 } & Adaptado & 6,66 & 6,38 & 6,67 & 6,56 \\
\cline { 2 - 6 } & Total & 6,05 & 6,98 & 6,71 & 6,63 \\
\hline \multirow{2}{*}{$\begin{array}{l}\text { Diversidad } \\
\text { Verbal }\end{array}$} & Original &, 12 &, 16 &, 16 &, 14 \\
\cline { 2 - 6 } & Adaptado &, 15 &, 19 &, 18 &, 17 \\
\cline { 2 - 6 } & Total &, 12 &, 16 &, 16 &, 15 \\
\hline
\end{tabular}

Las figuras 9 a 12 muestran las diferencias entre las medias en cada Nivel de dificultad para ambos Tipos de textos: lecturas originales en la fila superior y color más oscuro, lecturas adaptadas en la fila inferior y color claro. 


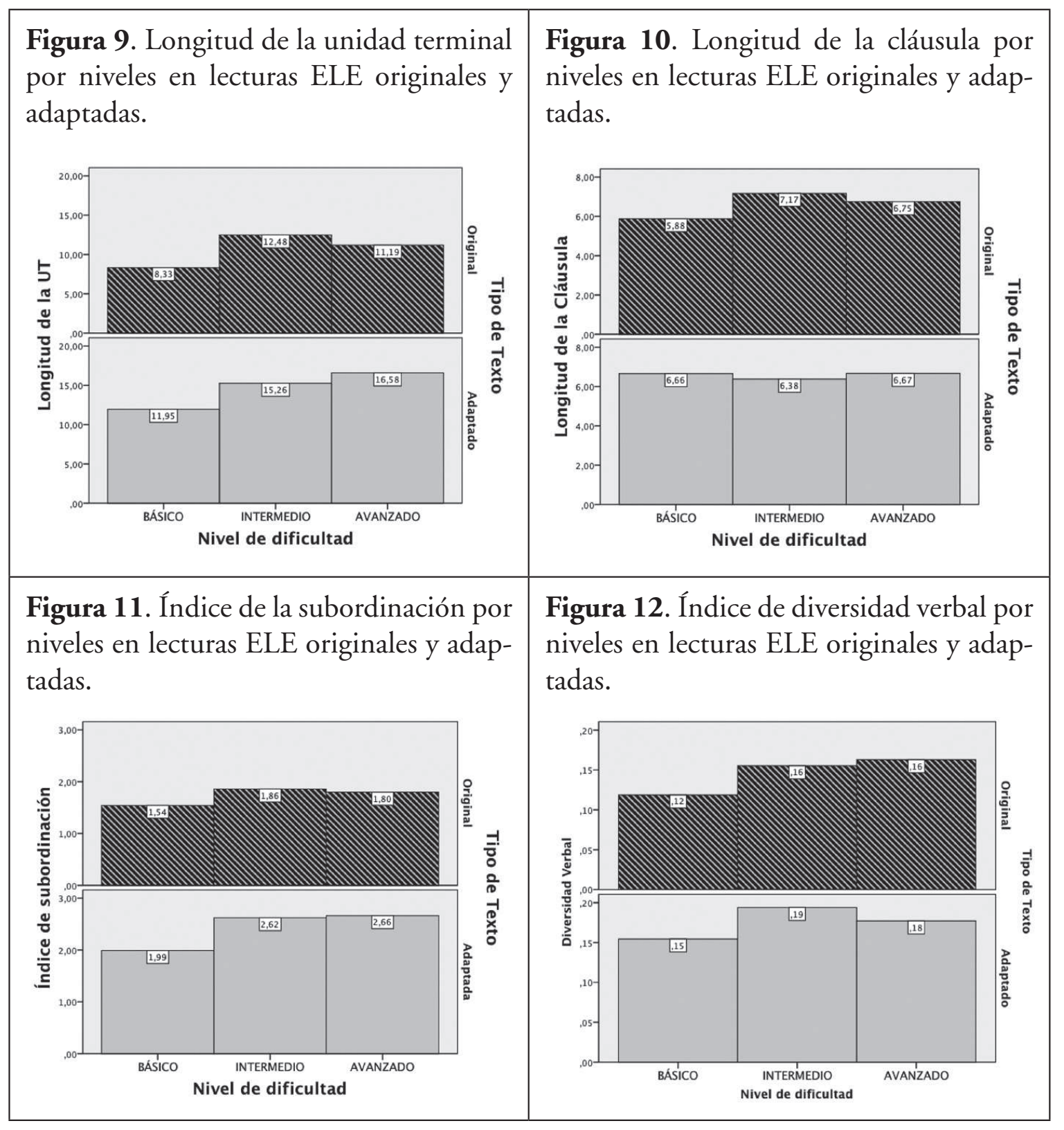

El comportamiento por niveles resulta diferente para todos los índices de elaboración morfosintáctica en los dos tipos de textos. De nuevo la Longitud de la UT y el Índice de subordinación muestran la misma variación entre Niveles de dificultad y Tipo de texto. En los textos originalmente escritos para ELE el nivel intermedio es el que alcanza mayor complejidad, aunque está muy cercano al nivel avanzado. Por el contrario, en los textos adaptados, el crecimiento es constante, aunque de nuevo hay mayor diferencia entre el nivel básico y el intermedio que entre el intermedio y el avanzado. Lo contrario ocurre para el Índice de diversidad verbal, pues en los textos adaptados decrece del nivel intermedio al avanzado, mientras en los originales se incrementa. Finalmente, la Longitud de la cláusula se mantiene similar en los tres niveles en los textos adaptados, pero decrece en el 
intermedio. En cambio, en los textos originales se observa la tendencia opuesta, y el nivel intermedio presenta la mayor Longitud de cláusula, si bien en el nivel avanzado es también bastante más complejo que en el inicial. Necesitamos ahora realizar algunas pruebas de diferencias para decidir si las diferencias son significativas. La Tabla VIII resume los resultados de aplicar las correspondientes pruebas estadísticas de significatividad:

Tabla VIII. Significatividad de diferencias (valores p) de los índices de complejidad morfosintáctica según el Nivel de dificultad en cada uno de los Tipos de texto.

\begin{tabular}{|l|c|c|c|c|}
\hline $\begin{array}{l}\text { Significatividad } \\
\text { de Niveles }\end{array}$ & $\begin{array}{c}\text { Longitud } \\
\text { de la UT }\end{array}$ & $\begin{array}{c}\text { Longitud } \\
\text { de la cláusula }\end{array}$ & $\begin{array}{c}\text { Índice de } \\
\text { Subordinación }\end{array}$ & $\begin{array}{c}\text { Diversidad } \\
\text { Verbal }\end{array}$ \\
\hline Originales & $\mathbf{0 , 0 0 0}$ & $\mathbf{0 , 0 1 4}$ & $\mathbf{0 , 0 0 4}$ & $\mathbf{0 , 0 0 6}^{*}$ \\
\hline Adaptados & $0,115^{*}$ & $0,724^{*}$ & $0,100^{*}$ & $0,650^{*}$ \\
\hline
\end{tabular}

Las pruebas de significatividad de diferencias revelan que éstas nunca son significativas en los textos adaptados y sin embargo siempre lo son en los textos originales, incluso para la Longitud de la cláusula.

En los textos originales, cuyas diferencias entre niveles de dificultad sí son significativas, los tres índices de elaboración sintáctica aumentan hasta el nivel intermedio, pero disminuyen un poco después. En el caso de la diversidad verbal, ésta aumenta a lo largo de los tres niveles, aunque más pronunciadamente entre el nivel básico y el intermedio que entre el intermedio y el avanzado. Las tendencias, no significativamente diferentes, en los textos adaptados exhiben otro comportamiento, como veíamos al discutir las figuras 9 a 12.

Por tanto, aunque los valores de los índices de los fragmentos adaptados son casi siempre mayores que los de sus homólogos originales, las diferencias entre niveles no son significativas y tampoco siguen todos el mismo desarrollo por niveles. ¿A qué pueden obedecer estas diferencias entre los textos adaptados y los originales? Una observación más atenta a los datos de la desviación típica en la Tabla $\mathrm{V}$ revela información significativa sobre valores más extremos en algunos de los índices. En general, salvo para la Longitud de la cláusula, en todos los índices se aprecia una dispersión mayor en los textos adaptados que en los originales. Así, la desviación típica del Índice de subordinación es mucho más alta en los textos adaptados, 0.83 (un $42.77 \%$ del valor de la media 1.95) frente a 0.29 de los originales (un 15.04\%). La Longitud de la UT se desvía típicamente un $37.53 \%$ de la media en los textos adaptados frente al $27.57 \%$ en los textos originales. La desviación estándar es similar entre los dos tipos de texto para la Diversidad verbal. 
Las figuras 16 a 19 presentan la dispersión de cada índice, según el tipo de texto y para cada nivel de dificultad?

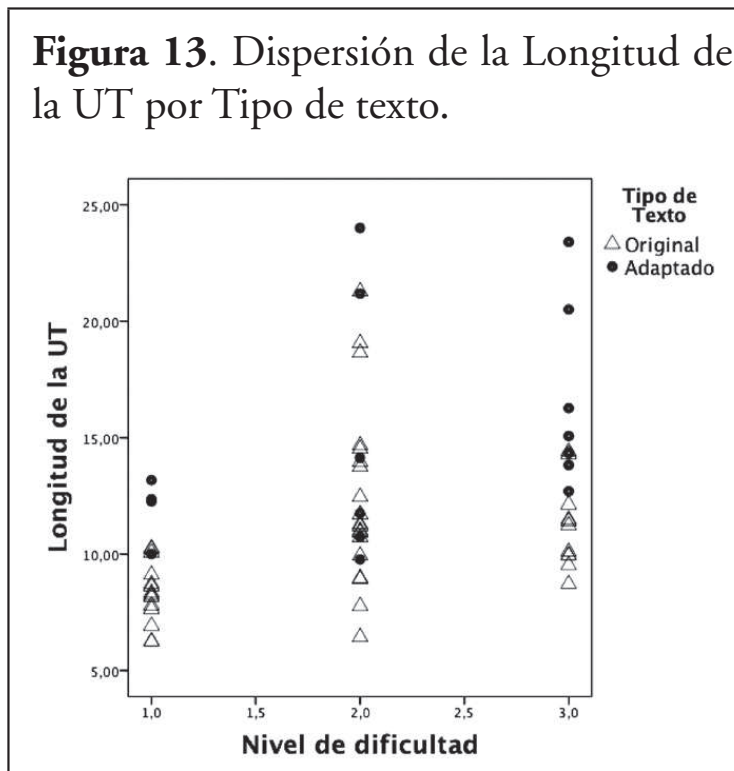

Figura 15. Dispersión del Índice de subordinación por Tipo de texto.

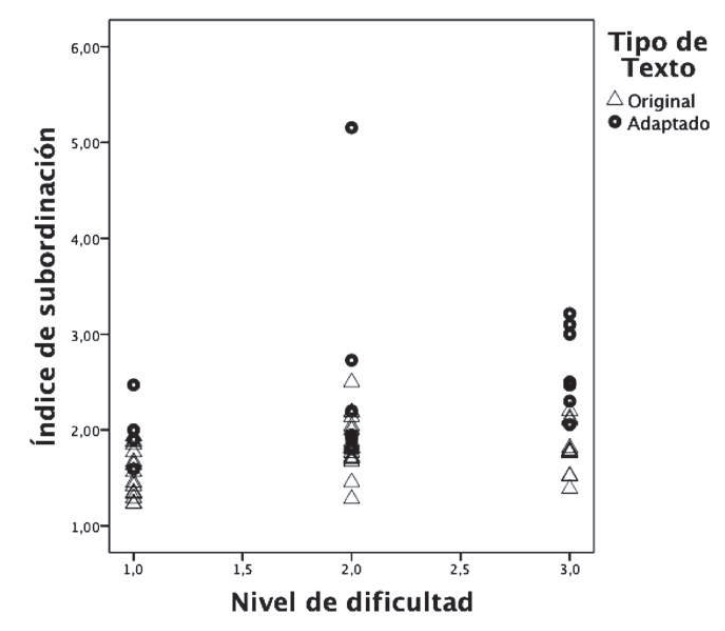

Figura 14. Dispersión de la Longitud de la cláusula por Tipo de texto.

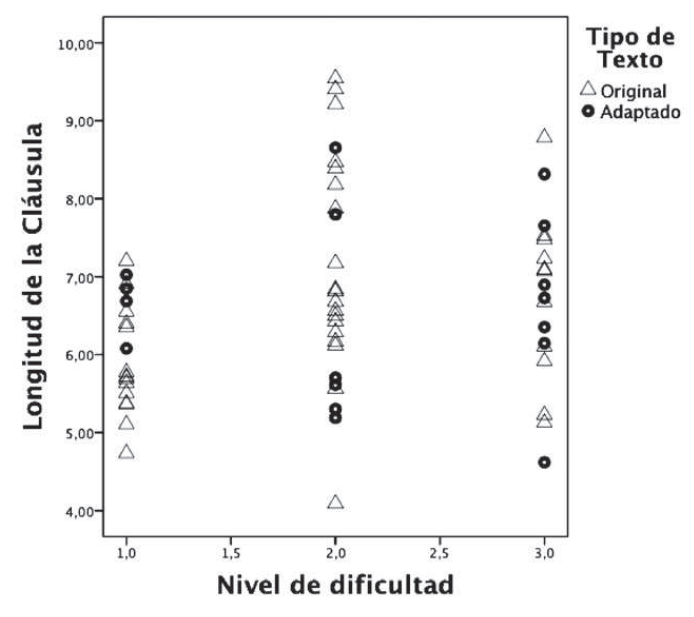

Figura 16. Dispersión de la Diversidad verbal por Tipo de texto.

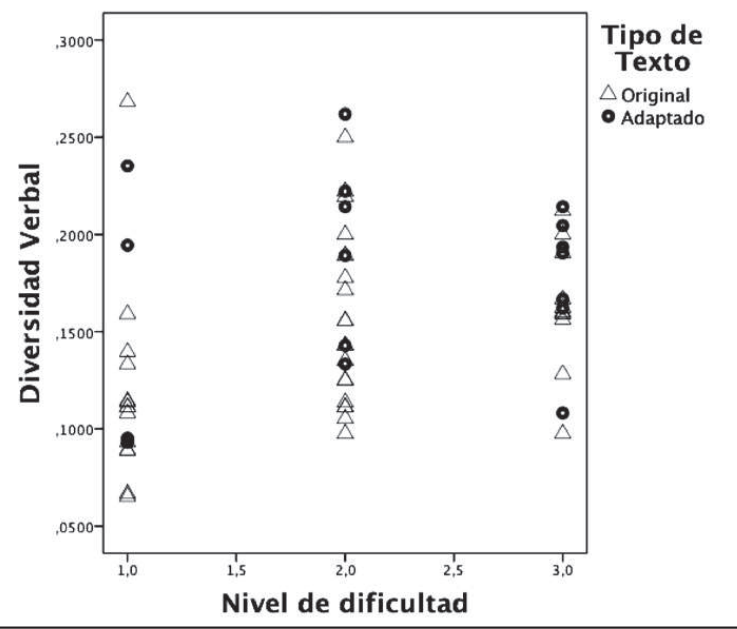

En estos gráficos se puede apreciar que los valores de los índices en los textos adaptados están más dispersos, en particular los valores altos, en la Longitud de la UT y el Índice de subordinación. En qué niveles ocurre más, es diferente para cada índice. En el caso de la Longitud de la cláusula, por ejemplo, hay una similar dispersión de los datos en ambos tipos de textos. De estas dispersiones podemos concluir tentativamente que es posible que los valores de los textos adaptados sean más erráticos, menos consistentes, y tal vez esto sea lo que haga que las diferencias

\footnotetext{
${ }^{9}$ Nivel 1,0 = Nivel básico; Nivel 2,0= Nivel intermedio; Nivel 3,0= Nivel avanzado.
} 
entre los diferentes niveles de dificultad no sean significativas en este tipo de texto, al ser más difícil encontrar tendencias centrales para los textos adaptados.

\subsection{Correlaciones entre los índices de complejidad morfosintáctica}

Observemos ahora las correlaciones entre los índices de complejidad morfosintáctica para ver si éstos se relacionan entre sí. En principio, la Longitud de la UT debería correlacionarse fuertemente con el Índice de subordinación (si las oraciones se hacen más complejas añadiendo más cláusulas) y con la Longitud de la cláusula (si las oraciones se hacen más complejas añadiendo más elementos intraclausales). Las correlaciones entre las variables dependientes pueden observarse en la Tabla IX.

Tabla IX. Correlaciones entre las variables dependientes.

\begin{tabular}{|l|c|c|c|c|}
\hline $\begin{array}{l}\text { Correlación } \\
\text { (Pearson) }\end{array}$ & $\begin{array}{c}\text { Longitud } \\
\text { de la UT }\end{array}$ & $\begin{array}{c}\text { Longitud } \\
\text { de la cláusula }\end{array}$ & $\begin{array}{c}\text { Índice de } \\
\text { subordinación }\end{array}$ & $\begin{array}{c}\text { Diversidad } \\
\text { verbal }\end{array}$ \\
\hline Longitud de la UT & 1 &, $523^{* *}$ &, $799^{* *}$ &, $367^{* *}$ \\
\hline Longitud de la cláusula &, $523^{* *}$ & 1 &,- 014 &,- 022 \\
\hline Índice de subordinación &, $799^{* *}$ &,- 014 & 1 &, $409^{* *}$ \\
\hline Diversidad verbal &,$- 404^{* *}$ &,- 022 &,$- 409^{* *}$ & 1 \\
\hline
\end{tabular}

${ }^{* *}$ Correlaciones significativas al 0,01 .

Existe una correlación positiva y significativa entre, sobre todo, el Índice de subordinación y la Longitud de la UT, lo cual no sorprende teniendo en cuenta su similar comportamiento en los diferentes niveles de dificultad y tipos de textos, así como la similar significatividad que ambos exhiben. También hay una clara, aunque no tan alta, correlación entre la Longitud de la cláusula y la Longitud de la UT. Así, la Longitud de la UT parece aumentar sobre todo por la añadidura de oraciones subordinadas, pero también por cierta mayor complejidad dentro de la cláusula. La Diversidad verbal se correlaciona con la Longitud de la UT, y un poco más con el Índice de subordinación, y no se correlaciona con la Longitud de la cláusula. Es decir, es fundamentalmente cuando se añade más subordinación (alargando así la UT) cuando se produce más diversidad verbal, si bien, dado que la correlación no es muy alta, es sólo una de las razones. 


\section{DISCUSIÓN DE LOS RESULTADOS}

Con respecto a la pregunta inicial acerca de la complejidad morfosintáctica de lecturas ELE de diferente dificultad, sí se observa una variación de la misma conforme aumenta el nivel de dificultad en el que la editorial clasifica cada texto, pero esta diferencia es más notable entre el nivel básico vs. niveles intermedio y avanzado, que entre los dos niveles más avanzados, puesto que estos últimos niveles no se diferencian significativamente, sino sólo con respecto al básico, para todos los índices salvo la Longitud de la cláusula, que no resultó significativa. Otro síntoma de que los textos no aumentan significativamente su complejidad morfosintáctica a partir del nivel intermedio es que en los textos originales, donde la diferencia de medias es altamente significativa estadísticamente, los índices primarios de complejidad sintáctica decrecen del nivel intermedio al avanzado. La Diversidad verbal sí aumenta entre el nivel intermedio y el avanzado, pero muy ligeramente. Por tanto, parece que las lecturas ELE exhiben características claramente más simples en un primer nivel, pero la elaboración deja de crecer significativamente después del nivel intermedio. Esto se aprecia fundamentalmente en los textos originales, ya que para los textos adaptados las diferencias no son significativas.

Acerca de la variación de los diferentes índices, los dos índices que varían más según la dificultad del texto son la Longitud de la UT y el Índice de subordinación, haciéndolo de forma continuada aunque como hemos comentado fundamentalmente entre el primer nivel y el resto de niveles. El crecimiento de la Diversidad verbal también es consistente con el Nivel de dificultad y significativo estadísticamente. Sin embargo, la Longitud de la cláusula decrece entre nivel intermedio y el avanzado, y las diferencias entre niveles no son significativas. Así pues, la Longitud de la UT crece debido fundamentalmente a un aumento de la cantidad de subordinación y no tanto debido a hacer más compleja la cláusula, añadiendo complementos a la misma o alargando los existentes. Esto queda patente también en el estudio de correlación, puesto que la Longitud de la cláusula se correlaciona menos que el Índice de subordinación con la Longitud de la UT. Por otro lado, la Diversidad verbal aumenta con la subordinación, como se observa en su correlación, lo que es de esperar debido a que la subordinación en español requiere con frecuencia otros tiempos - por la consecutio temporum - u otro modo -por ser el modo subjuntivo marca de subordinación, así como diversas formas impersonales del verbo a veces. En definitiva, parece que los textos se hacen más simples sobre todo por una menor subordinación que redunda en una Longitud de la UT menor también. Son estos dos índices, Índice de subordinación y Longitud de la UT, los que mejor reflejan ciertas diferencias en la elaboración morfosintáctica de los varios niveles de dificultad de las lecturas. La Longitud de la cláusula es el índice menos afectado por el nivel de dificultad (significativamente sólo para los textos originales). La Diversidad verbal se sitúa en un punto intermedio en cuanto a su 
relación con la simplificación de lecturas ELE, más próximo a la Longitud de la UT y el Índice de subordinación especialmente.

En cuanto a la distinción entre textos adaptados y originales, ¿se justifica dicha distinción por un comportamiento diferente de la complejidad sintáctica por niveles de dificultad? Los resultados indican que efectivamente cada tipo de texto tiene un comportamiento diferente. En primer lugar, las diferencias en elaboración morfosintáctica sólo son significativas para el caso de los textos originales. La tendencia de crecimiento es además diferente, puesto que mientras los textos originales en general aumentan significativamente del nivel básico al intermedio y disminuyen luego entre el nivel intermedio y el avanzado, salvo para el Índice de diversidad verbal que sigue creciendo en el nivel avanzado aunque muy levemente, los índices se comportan de forma más errática en los textos adaptados. Longitud de la UT e Índice de subordinación crecen de forma constante, la Longitud de la cláusula decrece del nivel básico al intermedio y vuelve a crecer en el avanzado hasta un nivel similar al nivel en la dificultad básica, mientras la Diversidad verbal se comporta de modo opuesto, con su valor máximo en el nivel intermedio. El comportamiento un tanto errático de la Longitud de la cláusula en los textos adaptados, junto con la muy pequeña diferencia entre niveles, parece estar detrás de que este índice no sea significativo para el conjunto del corpus.

Varias pueden ser las razones para las diferencias por Tipo de texto. Se observa una dispersión mayor en los textos adaptados que en los originales, especialmente en la Longitud de la unidad terminal y el Índice de subordinación, lo que podría deberse al menor número de fragmentos de este tipo en el muestreo o a factores intrínsecos al proceso de adaptación. Es posible que la complejidad sintáctica en el original juegue un papel en el producto de una versión simplificada, o que diferentes adaptadores se apeguen más o menos a los textos originales. Sea como fuere, el lector que elige leer un libro adaptado, en lugar de un libro escrito originalmente para aprendices de español, no puede esperar que la diferencia entre los varios niveles de dificultad se refleje morfosintácticamente, de modo que un texto de nivel básico puede tener oraciones o cláusulas tan largas y complejas como un texto de nivel avanzado, e igualmente con la diversidad morfológica verbal.

\section{CONCLUSIONES}

Las personas que escriben o editan textos para aprendices de español como lengua extranjera tienden a reducir la complejidad sintáctica de estos textos, pero parecen hacerlo de forma más consistente en el caso de los textos escritos originalmente como lecturas para ELE. Entre los métodos para simplificar la complejidad morfosintáctica, el más empleado es el uso de la parataxis y la reducción del número de cláusulas subordinadas, que lleva aparejada una disminución de la diversidad 
verbal. La cláusula no varía significativamente en líneas generales, por lo que parece que su simplificación se siente menos necesaria. Crossley, Louwerse, McCarthy y McNamara (2007) encontraron que los textos nativos tenían una Longitud de cláusula menor que los textos no-nativos. Tal vez esto se debiera en su caso al intento de simplificar las oraciones complejas usando complementos nominales en lugar de cláusulas subordinadas. Si esto ocurriera también en español se podría tener una primera causa para una Longitud de la cláusula más o menos constante por niveles de dificultad: la cláusula comienza desde un principio a ser más larga como recurso para evitar la subordinación, aunque de hecho, al aumentar la subordinación, la Longitud de la cláusula no disminuye. Otra causa más plausible quizás es que los complementos del nombre se perciben como más rápidamente adquiridos, o más intuitivamente -de hecho, en la mayoría de manuales o libros de textos apenas se explican. Así, aunque una cláusula más extensa probablemente ralentice la lectura, no la hará imposible o confusa casi nunca, dentro, asumimos, de un límite. Por fin, combinando las dos explicaciones, es posible que se comience, ya en el nivel básico, con una Longitud de la cláusula relativamente alta, al no percibirse demasiado complicada, pero al mismo tiempo, una mayor longitud constituiría un salto cualitativo en la dificultad de comprensión, por lo que no se va mucho más allá de la Longitud de cláusula del nivel intermedio, y se mantiene casi igual en el avanzado o incluso baja.

Un hecho a destacar es que parece ser más fácil simplificar mucho que hacerlo para un nivel intermedio, que se distingue menos del nivel avanzado que del básico. En este sentido, hay cierto trabajo por hacer para encontrar un nivel realmente intermedio de dificultad morfosintáctica.

Finalmente, los textos adaptados presentan más diversidad en su complejidad morfosintáctica y la diferencia entre niveles no es significativa, lo que podría deberse a varios factores, como por ejemplo la diversidad de la complejidad morfosintáctica de los propios textos fuente que se están adaptando, o la diferencia en el grado de apego al texto original de los diversos adaptadores, o tal vez la mayor o menor necesidad de adaptar el texto, por ejemplo por ser éste antiguo o moderno, donde los que necesitan de mayor adaptación -y por tanto a los que el adaptador no puede estar tan apegado- pueden encontrar su complejidad sintáctica más reducida.

El estudio de la complejidad morfosintáctica de las lecturas de ELE, por tanto, revela que hay una graduación por niveles de dificultad, pero sobre todo entre el nivel básico frente a intermedio y avanzado y sólo en los textos originales de manera significativa.

En futuros estudios sería conveniente estudiar también la relación de otros factores sintácticos y discursivos que no son únicamente la complejidad morfosintáctica. Trabajos recientes (Oh, 2001; Crossley, Greenfield y McNamara, 2008; Geva, 1992) han demostrado que medidas de correferencialidad, conectores, ob- 
viedad y relevancia de la estructura usada y similares variables están correlacionadas con una mejor comprensión de los textos en una L2. También sería interesante observar la interacción entre la complejidad morfosintáctica y la variedad y dificultad del vocabulario, según su frecuencia absoluta en la lengua y, si fuera posible, también según su disponibilidad (cfr. López Morales, 1999, entre otros), de forma similar al estudio de Barnett (1986), pero definiendo la dificultad del vocabulario según otros parámetros, a nuestro entender más significativos y precisos ${ }^{10}$. Quedará entonces por determinar si estas diferencias de elaboración morfosintáctica se correlacionan con los niveles psicológicos de dificultad, esto es, si lecturas morfosintácticamente más complejas resultan más difíciles y hasta qué punto para los lectores no nativos de textos ELE. Esto sólo se podría lograr con experimentos de comprensión con input manipulado para estas variables.

\section{REFERENCIAS}

Barnett, Marva A. (1986). Syntactic and lexical/semantic skill in foreign language reading: Importance and interaction. The Modern Language Journal, 70 (4), 343-349.

Barry, Susan y Lazarte, Alejandro. (1995). Embedded clause effects on recall: Does high prior knowledge of content domain overcome syntactic complexity in students of Spanish? The Modern Language Journal, 79 (4), 491-504.

Blau, Eileen K. (1982). The effect of syntax on readability for ESL students in Puerto Rico. TESOL Quarterly, 16 (4), 517-526.

Brown, Roger. (1987). A comparison of the comprehensibility of modified and unmodified materials for ESL. University of Hawaii Working Papers in ESL, 6, 49-79.

Chaudron, Craig. (1983). Simplification of input: Topic reinstatements and their effects on L2 learners' recognition and recall. TESOL Quarterly, 17 (3), 437458.

Checa-Garcia, Irene. (2005a). Medidas del desarrollo sintáctico en adolescentes almerienses (12 a 18 años): Hitos descriptivos y aportaciones metodológicas. Almería: Servicio de Publicaciones de la Universidad de Almería.

Checa-Garcia, Irene. (2005b). Medidas de madurez sintáctica aplicadas a lecturas de ELE. Interlingüistica, 16, 273-285.

Choi, Y.-J. (1996). A study of the relationship of cultural schema and linguistic complexity to the learners' reading comprehension in EFL. Tesis de magistra-

${ }^{10}$ Barnett simplifica el vocabulario de los textos eliminando las palabras más largas (generalmente menos disponibles y adquiridas más tarde en el inglés) y aquellas que le parecen, como usuaria, más difíciles. 
tura. Seúl, Korea: Universidad Nacional de Seúl.

Crossley, Scott A., Greenfield, Jerry y McNamara, Danielle S. (2008). Assessing Text Readability Using Cognitively Based Indices. TESOL Quarterly, 42 (3), 475-493.

Crossley, Scott A., Louwerse, Max L., McCarthy, Phillip M. y McNamara, Danielle S. (2007). A linguistic analysis of simplified and authentic texts. The Modern Language Journal, 91 (1), 15-30.

Crowhurst, Marion. (1980). Syntactic complexity in narration and argument at three grade levels. Canadian Journal of Education, 5, 6-13.

Crowhurst, Marion y Piche, Gene. (1979). Audience and mode of discourse effects on syntactic complexity in writing at two grade levels. Research in the Teaching of English, 13, 101-109.

Espinet de Jesús, Lidia. (1992). Índices de madurez sintáctica en escritores profesionales puertorriqueños. Tesis doctoral. Universidad de Puerto Rico.

Espinet de Jesús, Lidia. (1996). Índices primarios de madurez sintáctica en escritores profesionales puertorriqueños: variables sexo y modo del discurso. REALE, 6, 37-52.

Flahive, Douglas E. y Snow, Beckie G. (1980). Measures of syntactic complexity in evaluating ESL compositions. En J. Oller y K. Perkings (eds.) Research in language testing. (171-176). Rowley, MA: Newbury House.

Gaies, Stephen. (1979). Linguistic input in formal second language learning: The issue of syntactic gradation and readability in ESL materials. TESOL Quarterly, 13 (1), 41-50.

Gaies, Stephen. (1980). T-unit analysis in second language research: Applications, problems and limitations. TESOL Quarterly, 14 (1), 53-60.

Geva, Esther. (1992). The role of conjunctions in L2 text comprehension. TESOL Quarterly, 26 (4), 731-747.

Giles, Howard y Ogay, Tania. (2007). Communication accommodation theory. En B. Whaley y W. Samter (eds.), Explaining communication: Contemporary theories and exemplars (pp. 325-344). Mahwah, NJ: Lawrence Erlbaum.

Hunt, Kellog W. (1965). Grammatical structures written at three grade levels (Research Report $N^{o}$ 3). Urbana Champaign [Ill]: National Council of Teachers of English.

Hunt, Kellog W. (1967). Sentence structures used by superior students in grades four and twelve, and by superior adults. Tallahassee [Fl]: Florida State University.

Hunt, Kellog W. (1970a). Syntactic maturity in schoolchildren and adults. (Monographs of the Society for Research in Child Development No 134, vol. $35 \mathrm{~N}^{\circ}$ 1). Tallahassee, [Fl]: Florida State University.

Hunt, Kellog W. (1970b). Recent measures in syntactic development. En M. Lester (ed.), Readings in applied transformational grammar (pp. 193-201). New York: Holt, Rinehart y Winston. 
Jeong, Y.-K. (1987). The effect of syntactic simplification on Korean students' English reading comprehension. Tesis de magistratura. Seúl, Korea: Universidad Nacional de Seúl.

Kim, Y.-J. (1985). Variables in reading comprehension. Tesis de magistratura. Seúl, Korea: Universidad Nacional de Seúl.

Krashen, Stephen D. (1982). Principles and practice in second language acquisition. Oxford: Pergamon.

Larsen-Freeman, Diane. (1978). An ESL index of development. TESOL Quaterly, $12,439-448$.

Larsen-Freeman, Diane y Strom, Virginia. (1977). The construction of a second language acquisition index of development. Language Learning, 27, 123-134.

Lee, James F. (1998). The relationship of verb morphology to second language reading comprehension and input processing. The Modern Language Journal, 82 (1), 33-48.

Loban, Walter. 1976. Language development: Kindergarten through grade twelve (Research Report No 18). Urbana [Ill]: National Council of Teachers of English.

Long, M. H. (1985). Input and second language acquisition theory. En S. M. Gass y C. G. Madden (eds.) Input in second language acquisition (pp. 377-393). Rowley, MA: Newbury House.

López Morales, Humberto. (1999). Léxico disponible de Puerto Rico. Madrid: Arco Libros.

MacNamara, John T. (1967). Comparative studies of reading and problem solving in two languages. Montreal: Mc Gill University (Language Research Group).

Mendelsohn, David J. (1983). The case for considering syntactic maturity in E.S.L. and E.F.L. International Review of Applied Linguistics, 21 (4), 299-311.

Nippold, Marylin A. (1998). Later language development. The school-age and adolescent years. Austin, TX: Pro-ed.

Oh, Sun-Young. (2001). Two types of input modification and EFL reading comprehension: simplification versus elaboration. TESOL Quarterly, 35 (1), 6996.

Prater, Doris y Padia, William. (1983). Effects of modes of discourse on writing performance in grades four and six. Research in the Teaching of English, 17, 127-134.

Rodrigues, Raymond J. (1981). A longitudinal study of bilingual English syntax. Aztlán, 12 (1), 75-87.

Rodríguez Fonseca, Leonilda. (1991). Índices de madurez sintáctica en escolares puertorriqueños de escuela primaria. En H. López Morales (ed.), La enseñanza del español como lengua materna (pp. 133-143). Actas del II Seminario Internacional sobre Apuntes de la Lingüística a la Enseñanza del Español como Lengua Materna. Río Piedras: Puerto Rico. 
Torres González, Antonia N. (1996). Complejidad sintáctica en estudiantes de Tenerife. Santa Cruz de Tenerife: Centro de la Cultura Popular Canaria.

Torres González, Antonia N. (1997). Complejidad sintáctica en escritores de las Islas Canarias. REALE, 8, 105-125.

Véliz de Vos, Mónica. (1999). Complejidad sintáctica y modo del discurso. Estudios Filológicos, 34, 181-190. 